\title{
HARMONIC ANALYSIS ON THE QUOTIENT SPACES OF HEISENBERG GROUPS
}

\author{
JAE-HYUN YANG
}

A certain nilpotent Lie group plays an important role in the study of the foundations of quantum mechanics ([Wey]) and of the theory of theta series (see [C], [I] and [Wei]). This work shows how theta series are applied to decompose the natural unitary representation of a Heisenberg group.

For any positive integers $g$ and $h$, we consider the Heisenberg group

$$
H_{R}^{(g, h)}:=\left\{[(\lambda, \mu), \kappa] \mid \lambda, \mu \in R^{(h, g)}, \kappa \in R^{(h, h)}, \kappa+\mu^{t} \lambda \text { symmetric }\right\}
$$

endowed with the following multiplication law

$$
[(\lambda, \mu), \kappa] \circ\left[\left(\lambda^{\prime}, \mu^{\prime}\right), \kappa^{\prime}\right]=\left[\left(\lambda+\lambda^{\prime}, \mu+\mu^{\prime}\right), \kappa+\kappa^{\prime}+\lambda^{t} \mu^{\prime}-\mu^{t} \lambda^{\prime}\right] .
$$

The mapping

$$
H_{R}^{(g, h)} \ni[(\lambda, \mu), \kappa] \longrightarrow\left(\begin{array}{cccc}
E_{g} & 0 & 0 & { }^{t} \mu \\
\lambda & E_{h} & \mu & \kappa \\
0 & 0 & E_{g} & -{ }^{t} \lambda \\
0 & 0 & 0 & E_{h}
\end{array}\right)
$$

defines an embedding of $H_{R}^{(g, h)}$ into the symplectic group $S p(g+h, R)$. We refer to [Z] for the motivation of the study of this Heisenberg group $H_{R}^{(g, h)} . H_{Z}^{(g, h)}$ denotes the discrete subgroup of $H_{R}^{(g, h)}$ consisting of integral elements, and $L^{2}\left(H_{Z}^{(g, h)} \backslash H_{R}^{(g, h)}\right)$ is the $L^{2}$-space of the quotient space $H_{Z}^{(g, h)} \backslash$ $H_{R}^{(g, h)}$ with respect to the invariant measure

$$
d \lambda_{11} \cdots d \lambda_{h, g-1} d \lambda_{h g} d \mu_{11} \cdots d \mu_{h, g-1} d \mu_{h g} d \kappa_{11} d \kappa_{12} \ldots d \kappa_{h-1, h} d \kappa_{h h} .
$$

We have the natural unitary representation $\rho$ on $L^{2}\left(H_{Z}^{(g, h)} \backslash H_{R}^{(g, h)}\right)$ given by

$$
\rho\left(\left[\left(\lambda^{\prime}, \mu^{\prime}\right), \kappa^{\prime}\right]\right) \phi([(\lambda, \mu), \kappa])=\phi\left([(\lambda, \mu), \kappa] \circ\left[\left(\lambda^{\prime}, \mu^{\prime}\right), \kappa^{\prime}\right]\right) .
$$

Received October 11, 1990. 
The Stone-von Neumann theorem says that an irreducible representation $\rho$ of $H_{R}^{(g, h)}$ is characterized by a real symmetric matrix $c \in R^{(h, h)}(c \neq 0)$ such that

$$
\rho_{c}([(0,0), \kappa])=\exp \{\pi i \sigma(c \kappa)\} I, \quad \kappa={ }^{t} \kappa \in R^{(h, h)},
$$

where $I$ denotes the identity mapping of the representation space. If $c=0$, then it is characterized by a pair $(k, m) \in R^{(h, g,} \times R^{(h, g)}$ such that

$$
\rho_{k, m}([(\lambda, \mu), \kappa])=\exp \left\{2 \pi i \sigma\left(k^{t} \lambda+m^{t} \mu\right)\right\} I .
$$

But only the irreducible representations $\rho_{\mathcal{N}}$ with $\mathscr{M}={ }^{t} \mathscr{M}$ even integral and $\rho_{k, m}\left(k, m \in Z^{(h, g)}\right)$ could occur in the right regular representation $\rho$ in $L^{2}\left(H_{Z}^{(g, h)} \backslash H_{R}^{(g, h)}\right)$.

In this article, we decompose the right regular representation $\rho$. The real analytic functions defined in (1.5) play an important role in decomposing the right regular representation $\rho$.

Notations. We denote $Z, R$ and $C$ the ring of integers, the field of real numbers and the field of complex numbers respectively. $F^{(k, l)}$ denotes the set of all $k \times l$ matrices with entries in a commutative ring $F E_{g}$ denotes the identity matrix of degree $g$. $\sigma(A)$ denotes the trace of a square matrix $A$.

$$
\begin{aligned}
Z_{\geq 0}^{(h, g)} & =\left\{J=\left(J_{k l}\right) \in Z^{(h, g)} \mid J_{k l} \geq 0 \text { for all } k, l\right\}, \\
|J| & =\sum_{k, l} J_{k l}, \\
J \pm \varepsilon_{k l} & =\left(J_{11}, \cdots, J_{k l} \pm 1, \cdots, J_{h g}\right), \\
(\lambda+N+A)^{J} & =\left(\lambda_{11}+N_{11}+A_{11}\right)^{J_{11}} \cdots\left(\lambda_{h g}+N_{h g}+A_{h g}\right)^{J_{h g}} .
\end{aligned}
$$

\section{§1. Theta series}

Let $H_{g}$ be the Siegel upper half plane of degree $g$. We fix an element $\Omega \in H_{g}$ once and for all. Let $\mathscr{M}$ be a positive definite, symmetric even integral matrix of degree $h$. A holomorphic function $f: C^{(h, g)} \rightarrow C$ satisfying the functional equation

$$
f(W+\lambda \Omega+\mu)=\exp \left\{-\pi i \sigma\left(\mathscr{M}\left(\lambda \Omega{ }^{t} \lambda+2 \lambda^{t} W\right)\right)\right\} f(W)
$$

for all $\lambda, \mu \in Z^{(h, g)}$ is called a theta series of level $\mathscr{M}$ with respect to $\Omega$. The set $T_{\alpha}(\Omega)$ of all theta series of level $\mathscr{M}$ with respect to $\Omega$ is a vector space of dimension $(\operatorname{det} \mathscr{M})^{g}$ with a basis consisting of theta series 


$$
\vartheta^{(\mathscr{M})}\left[\begin{array}{c}
A \\
0
\end{array}\right](\Omega, W):=\sum_{\left.N \in Z^{(n,}, \boldsymbol{g}\right)} \exp \left\{\pi i \sigma\left\{\mathscr{M}\left((N+A) \Omega^{t}(N+A)+2 W^{t}(N+A)\right)\right\}\right\},
$$

where $A$ runs over a complete system of representatives of the cosets $\mathscr{M}^{-1} Z^{(h, g)} / Z^{(h, g)}$.

Definition 1.1. A function $\varphi: C^{(h, g)} \times C^{(h, g)} \rightarrow C$ is called an auxiliary theta series of level $\mathscr{M}$ with respect to $\Omega$ if it satisfies the following conditions (i) and (ii):

(i) $\varphi(U, W)$ is a polynomial in $W$ whose coefficients are entire functions,

(ii) $\varphi(U+\lambda, W+\lambda \Omega+\mu)=\exp \left\{-\pi i\left(\mathscr{M}\left(\lambda \Omega^{t} \lambda+2 \lambda^{t} W\right)\right)\right\} \varphi(U, W)$ for all $(\lambda, \mu) \in Z^{(h, g)} \times Z^{(h, g)}$.

The space $\Theta_{\Omega}^{(\mathscr{A})}$ of all auxiliary theta series of level $\mathscr{M}$ with respect to $\Omega$ has a basis consisting of the following functions:

$$
\begin{aligned}
\vartheta_{J}^{(k)}\left[\begin{array}{c}
A \\
0
\end{array}\right](\Omega \mid \lambda, \mu+\lambda \Omega):=\sum_{N \in Z^{(h, g)}}(\lambda+N+A)^{J} \\
\quad \times \exp \left\{\pi i \sigma\left(\mathscr{M}\left((N+A) \Omega^{t}(N+A)+(\mu+\lambda \Omega){ }^{t}(N+A)\right)\right)\right\} .
\end{aligned}
$$

where $A$ (resp. $J$ ) runs over the cosets $\mathscr{M}^{-1} Z^{(h, g)} / Z^{(h, g)}$ (resp. $Z_{\geq 0}^{(h, g)}$ ).

Definition 1.2. A real analytic function $\varphi: R^{(h, g)} \times R^{(h, g)} \rightarrow C$ is called a mixed theta series of level $\mathscr{M}$ with respect to $\Omega$ if $\varphi$ satisfies the following conditions (1) and (2):

(1) $\varphi(\lambda, \mu)$ is a polynomial in $\lambda$ whose coefficients are entire functions in complex variables $Z=\mu+\lambda \Omega$;

(2) $\varphi(\lambda+\tilde{\lambda}, \mu+\tilde{\mu})=\exp \left\{-\pi i \sigma\left(\mathscr{M}\left(\tilde{\lambda} \Omega^{t} \tilde{\lambda}+2(\mu+\lambda \Omega){ }^{t} \tilde{\lambda}\right)\right)\right\} \varphi(\lambda, \mu)$ for all $(\tilde{\lambda}, \tilde{\mu}) \in Z^{(h, g)} \times Z^{(h, g)}$.

If $A \in \mathscr{M}^{-1} Z^{(h, g)} / Z^{(h, g)}$ and $J \in Z_{\geq 0}^{(h, g)}$,

$$
\begin{aligned}
\vartheta_{J}^{(\mu)}\left[\begin{array}{c}
A \\
0
\end{array}\right](\Omega \mid \lambda, \mu+\lambda \Omega):=\sum_{\left.N \in Z^{(h,}, g\right)}(\lambda+N+A)^{J} \\
\quad \times \exp \left\{\pi i \sigma\left(\mathscr{M}\left((N+A) \Omega^{t}(N+A)+2(\mu+\lambda \Omega)^{t}(N+A)\right)\right)\right\}
\end{aligned}
$$

is a mixed theta series of level $\mathscr{M}$.

Now for a positive definite symmetric even integral matrix $\mathscr{M}$ of degree $h$, we define a function on $H_{R}^{(g, h)}$.

$$
\begin{aligned}
\Phi_{J}^{(k)}\left[\begin{array}{c}
A \\
0
\end{array}\right](\Omega \mid[(\lambda, \mu), \kappa]):=\exp \left\{\pi i \sigma\left(\mathscr{M}\left(\kappa-\lambda^{t} \mu\right)\right)\right\} \sum_{\left.N \in Z^{(h,}, g\right)}(\lambda+N+A)^{J} \\
\\
\left.\times \exp \left\{\pi i \sigma\left(\mathscr{M}(\lambda+N+A) \Omega^{t}(\lambda+N+A)+2(\lambda+N+A)^{t} \mu\right)\right)\right\},
\end{aligned}
$$


where $A \in \mathscr{M}^{-1} Z^{(h, g)} / Z^{(h, g)}$.

Proposition 1.3.

$$
\begin{aligned}
\Phi_{J}^{(k)} & {\left[\begin{array}{c}
A \\
0
\end{array}\right](\Omega \mid[(\lambda, \mu), \kappa]) } \\
& =\exp \left\{2 \pi i \sigma\left(\mathscr{M} \mu^{t} A\right)\right\} \Phi_{J}^{(k)}\left[\begin{array}{l}
0 \\
0
\end{array}\right](\Omega \mid[(\lambda, \mu), \kappa] \circ[(A, 0), 0]) .
\end{aligned}
$$

$$
\begin{aligned}
\Phi_{J}^{(\mu)} & {\left[\begin{array}{c}
A \\
0
\end{array}\right](\Omega \mid[(\tilde{\lambda}, \tilde{\mu}), \tilde{\kappa}] \circ[(\lambda, \mu), \kappa])=\Phi_{J}^{(\mu)}\left[\begin{array}{c}
A \\
0
\end{array}\right](\Omega \mid[(\lambda, \mu), \kappa]) . } \\
& \left([(\tilde{\lambda}, \tilde{\mu}), \tilde{\kappa}] \in H_{Z}^{(h, g)},[(\lambda, \mu), \kappa] \in H_{R}^{(h, g)}, A \in \mathscr{M}^{-1} Z^{(h, g)} / Z^{(h, g)}\right) .
\end{aligned}
$$

Proof.

$$
\begin{aligned}
\Phi_{J}^{(\mu)}\left[\begin{array}{l}
0 \\
0
\end{array}\right]\left(\Omega \mid\left[(\lambda+A, \mu), \kappa-\mu^{t} A\right]\right) \\
=\exp \left\{\pi i \sigma\left(\mathscr{M}\left(\kappa-\mu^{t} A-(\lambda+A)^{t} \mu\right)\right)\right\} \sum_{N \in Z^{(h, g)}}(\lambda+A+N)^{J} \\
\\
\quad \times \exp \left\{\pi i \sigma\left(\mathscr{M}\left((\lambda+A+N) \Omega^{t}(\lambda+N+A)+2(\lambda+N+A)^{t} \mu\right)\right)\right\} \\
=\exp \left\{-2 \pi i \sigma\left(\mathscr{M}^{t} A\right)\right\} \Phi_{J}^{(\mu)}\left[\begin{array}{c}
A \\
0
\end{array}\right](\Omega \mid[(\lambda, \mu), \kappa]) .
\end{aligned}
$$

On the other hand, if $[(\tilde{\lambda}, \tilde{\mu}), \tilde{\kappa}] \in H_{Z}^{(h, g)}$,

$$
\begin{aligned}
\Phi_{J}^{(\mu)} & {\left[\begin{array}{c}
A \\
0
\end{array}\right](\Omega \mid[(\tilde{\lambda}, \tilde{\mu}), \tilde{\kappa}] \circ[(\lambda, \mu), \kappa]) } \\
= & \exp \left\{\pi i \sigma\left(\mathscr{M}\left(\tilde{\kappa}+\kappa+\tilde{\lambda}^{t} \mu-\tilde{\mu}^{t} \lambda-(\tilde{\lambda}+\lambda)^{t}(\tilde{\mu}+\mu)\right)\right)\right\} \sum_{N \in Z^{(h, g)}}(\tilde{\lambda}+\lambda+N+A)^{J} \\
& \times \exp \left\{\pi i \sigma\left((\tilde{\lambda}+\lambda+N+A) \Omega^{t}(\tilde{\lambda}+\lambda+N+A)+2(\tilde{\lambda}+\lambda+N+A)^{t}(\tilde{\mu}+\mu)\right)\right\} \\
= & \Phi_{J}^{(\mu)}\left[\begin{array}{c}
A \\
0
\end{array}\right](\Omega \mid[(\lambda, \mu), \kappa]) .
\end{aligned}
$$

Here in the last equality we used the facts that $\sigma\left(\mathscr{M}\left(\tilde{\kappa}-{ }^{t} \tilde{\lambda} \tilde{\kappa}\right)\right) \in 2 Z$ and $\sigma\left(\mathscr{M} A^{t} \tilde{\mu}\right) \in Z$.

Remark. Proposition 1.3 implies that $\Phi_{J}^{(\mu)}\left[\begin{array}{c}A \\ 0\end{array}\right](\Omega \mid[(\lambda, \mu), \kappa])\left(J \in Z_{\geq 0}^{(h, g)}\right)$ are real analytic functions on the quotient space $H_{Z}^{(g, h)} \backslash H_{R}^{(g, h)}$.

The following matrices

$$
X_{k l}^{0}:=\left(\begin{array}{cccc}
0 & 0 & 0 & 0 \\
0 & 0 & 0 & E_{k l}^{0} \\
0 & 0 & 0 & 0 \\
0 & 0 & 0 & 0
\end{array}\right), \quad 1 \leq k \leq l \leq h,
$$




$$
\begin{aligned}
\hat{X}_{i j}: & =\left(\begin{array}{cccc}
0 & 0 & 0 & { }^{t} E_{i j} \\
0 & 0 & E_{i j} & 0 \\
0 & 0 & 0 & 0 \\
0 & 0 & 0 & 0
\end{array}\right), \quad 1 \leq i \leq h, \quad 1 \leq j \leq g, \\
X_{i j}: & =\left(\begin{array}{cccc}
0 & 0 & 0 & 0 \\
E_{i j} & 0 & 0 & 0 \\
0 & 0 & 0 & -{ }^{t} E_{i j} \\
0 & 0 & 0 & 0
\end{array}\right), \quad 1 \leq i \leq h, \quad 1 \leq j \leq g
\end{aligned}
$$

form a basis of the Lie algebra $\mathscr{H}_{R}^{(g, h)}$ of the Heisenberg group $H_{R}^{(g, h)}$. Here $E_{k l}^{0}(k \neq l)$ and $h \times h$ symmetric matrix with entry $1 / 2$ where the $k$-th (or $l$-th) row and the $l$-th (or $k$-th) column meet, all other entries 0 , $E_{k k}^{0}$ is an $h \times h$ diagonal matrice with the $k$-th diagonal entry 1 and all other entries 0 and $E_{i j}$ is an $h \times g$ matrix with entry 1 where the $i$-th row and the $j$-th column meet, all other entries 0 . By an easy calculation, we see that the following vector fields

$$
\begin{aligned}
& D_{k l}^{0}=\frac{\partial}{\partial \kappa_{k l}}, \quad 1 \leq k \leq l \leq h, \\
& D_{m p}=\frac{\partial}{\partial \lambda_{m p}}-\left(\sum_{k=1}^{m} \mu_{k p} \frac{\partial}{\partial \kappa_{k m}}+\sum_{k=m+1}^{h} \mu_{k p} \frac{\partial}{\partial \kappa_{m k}}\right), \\
& \hat{D}_{m p}=\frac{\partial}{\partial \mu_{m p}}+\left(\sum_{k=1}^{m} \lambda_{k p} \frac{\partial}{\partial \kappa_{k m}}+\sum_{k=m+1}^{h} \lambda_{k p} \frac{\partial}{\partial \kappa_{m k}}\right),
\end{aligned}
$$

form a basis for the Lie algebra of left invariant vector fields on $H_{R}^{(g, h)}$.

Theorem 1.

$$
\begin{gathered}
D_{k l}^{0} \Phi_{J}^{(k)}\left[\begin{array}{c}
A \\
0
\end{array}\right](\Omega \mid[(\lambda, \mu), \kappa])= \\
\hat{D}_{m p} \Phi_{J}^{(k)}\left[\begin{array}{c}
A \\
0
\end{array}\right](\Omega \mid[(\lambda, \mu), \kappa])=2 \pi i \sum_{l=1}^{h} \mathscr{M}_{m l} \Phi_{J}^{(k)} \Phi_{J+\varepsilon_{l p}}^{(\mu)}\left[\begin{array}{c}
A \\
0
\end{array}\right](\Omega \mid[(\lambda, \mu), \kappa]), \\
D_{m p} \Phi_{J}^{(k)}\left[\begin{array}{c}
A \\
0
\end{array}\right](\Omega \mid[(\lambda, \mu), \kappa])=2 \pi i \sum_{l=1}^{h} \sum_{q=1}^{g} \mathscr{M}_{l m} \Omega_{p q} \Phi_{J+\varepsilon_{l q}(\mu)}^{(\mu)}\left[\begin{array}{c}
A \\
0
\end{array}\right](\Omega \mid[(\lambda, \mu), \kappa]) \\
+J_{m p} \Phi^{(\mu)}\left[\begin{array}{c}
A \\
0
\end{array}\right](\Omega \mid[(\lambda, \mu), \kappa]) . \\
(1 \leq k \leq l \leq h, 1 \leq m \leq h, 1 \leq p \leq g)
\end{gathered}
$$

Proof. (1.8) follows immediately from the definition of $\Phi_{J}^{(\alpha)}\left[\begin{array}{c}A \\ 0\end{array}\right](\Omega \mid$ $[(\lambda, \mu), \kappa])$. 


$$
\begin{aligned}
& \hat{D}_{m p} \Phi_{J}^{(\mu)}\left[\begin{array}{c}
A \\
0
\end{array}\right](\Omega \mid[(\lambda, \mu), \kappa]) \\
& =-\pi i \sum_{i=1}^{h} \mathscr{M}_{m l} \lambda_{l p} \Phi_{J}^{(k)}\left[\begin{array}{c}
A \\
0
\end{array}\right](\Omega \mid[(\lambda, \mu), \kappa]) \\
& +2 \pi i\left\{\pi i \sigma\left(\mathscr{M}\left(\kappa-\lambda^{t} \mu\right)\right)\right\} \sum_{\left.N \in Z^{i n, g}\right)}(\lambda+N+A)^{J} \sum_{l=1}^{n} \mathscr{M}_{m l}(\lambda+N+A)_{l p} \\
& \times \exp \left\{\pi i \sigma\left(\mathscr{M}\left((\lambda+N+A) \Omega^{t}(\lambda+N+A)+2(\lambda+N+A)^{t} \mu\right)\right)\right\} \\
& +\pi i \sum_{l=1}^{h} \mathscr{M}_{m l} \lambda_{l p} \Phi_{J}^{(\mu)}\left[\begin{array}{c}
A \\
0
\end{array}\right](\Omega \mid[(\lambda, \mu), \kappa]) \\
& =2 \pi i \sum_{l=1}^{h} \mathscr{M}_{m l} \Phi_{J+\epsilon_{l p}}^{(\lambda)}\left[\begin{array}{c}
A \\
0
\end{array}\right](\Omega \mid[(\lambda, \mu), \kappa]) .
\end{aligned}
$$

We compute

$$
\begin{aligned}
\frac{\partial}{\partial \lambda_{m p}} \Phi_{J}^{(\mu)} & {\left[\begin{array}{c}
A \\
0
\end{array}\right](\Omega \mid[(\lambda, \mu), \kappa]) } \\
= & -\pi i \sum_{k=1}^{h} \mathscr{M}_{k m} \mu_{k p} \Phi_{J}^{(\mu)}\left[\begin{array}{c}
A \\
0
\end{array}\right](\Omega \mid[(\lambda, \mu), \kappa]) \\
& +2 \pi i \sum_{k=1}^{h} \sum_{q=1}^{g} \mathscr{M}_{k m} \Omega_{p q} \Phi_{J+\varepsilon_{k q}}^{(\mu)}\left[\begin{array}{c}
A \\
0
\end{array}\right](\Omega \mid[(\lambda, \mu), \kappa]) \\
& +J_{m p} \Phi_{J \epsilon_{\epsilon_{p}}(k)}^{(k)}\left[\begin{array}{c}
A \\
0
\end{array}\right](\Omega \mid[(\lambda, \mu), \kappa]) \\
& +2 \pi i \sum_{k=1}^{n} \mathscr{M}_{k m} \mu_{k p} \Phi_{J}^{(\mu)}\left[\begin{array}{c}
A \\
0
\end{array}\right](\Omega \mid[(\lambda, \mu), \kappa]) .
\end{aligned}
$$

Therefore we obtain (1.8) and (1.10).

q.e.d.

Corollary 1.4.

$$
\left(D_{m p}-\sum_{q=1}^{g} \Omega_{p q} \hat{D}_{m q}\right) \Phi_{J}^{(k)}\left[\begin{array}{c}
A \\
0
\end{array}\right](\Omega \mid[(\lambda, \mu), \kappa])=J_{m p} \Phi_{J-\varepsilon_{m p}}^{(\lambda)}\left[\begin{array}{c}
A \\
0
\end{array}\right](\Omega \mid[(\lambda, \mu), \kappa]) .
$$

Let $H_{\Omega}^{(\Omega)}\left[\begin{array}{l}A \\ 0\end{array}\right]$ be the completion of the vector space spanned by $\Phi_{J}^{(\Omega)}\left[\begin{array}{l}A \\ 0\end{array}\right](\Omega \mid$ $[(\lambda, \mu), \kappa])\left(J \in Z_{\Sigma}^{(h, g)}\right)$ and let $\overline{H_{\Omega}^{(k)}\left[\begin{array}{l}A \\ 0\end{array}\right]}$ be the complex conjugate of $H_{\Omega}^{(k)}\left[\begin{array}{c}A \\ 0\end{array}\right]$.

THEOREM 2. $H_{a}^{(k)}\left[\begin{array}{c}A \\ 0\end{array}\right]$ and $\overline{H_{a}^{(\alpha)}\left[\begin{array}{c}A \\ 0\end{array}\right]}$ are irreducible invariant subspaces of $L^{2}\left(H_{Z}^{(h, g)} \backslash H_{R}^{(h, g)}\right)$ with respect to the right regular representation $\rho$. In addition, we have 


$$
\begin{aligned}
& H_{\Omega}^{(.)}\left[\begin{array}{l}
A \\
0
\end{array}\right]=\exp \left\{2 \pi i \sigma\left(\mathscr{M} \mu^{t} A\right)\right\} H_{\Omega}^{(*)}\left[\begin{array}{l}
0 \\
0
\end{array}\right] \\
& \rho([(0,0), \tilde{\kappa}]) \phi=\exp \{\pi i \sigma(\mathscr{M} \tilde{\kappa})\} \phi \quad\left(\phi \in H_{\Omega}^{(\mathscr{k})}\left[\begin{array}{c}
A \\
0
\end{array}\right]\right), \\
& \rho([(0,0), \tilde{\kappa}]) \bar{\phi}=\exp \{-\pi i \sigma(\mathscr{M} \tilde{\kappa})\} \bar{\phi} \quad\left(\bar{\phi} \in \overline{H_{\Omega}^{(\mathscr{M})}\left[\begin{array}{c}
A \\
0
\end{array}\right]}\right) .
\end{aligned}
$$

Proof. It follows from Theorem 1, Proposition 1.3 and the definition of $\Phi_{J}^{(k)}\left[\begin{array}{c}A \\ 0\end{array}\right](\Omega \mid[(\lambda, \mu), \kappa])$.

q.e.d.

\section{§ 2. Proof of the Main Theorem}

We fix an element $\Omega \in H_{g}$ once and for all. We introduce a system of complex coordinates with respect to $\Omega$ :

$$
Z=\mu+\lambda \Omega, \quad \bar{Z}=\mu+\lambda \bar{\Omega}, \quad \lambda, \mu \text { real } .
$$

We set

$$
d Z=\left(\begin{array}{ccc}
d Z_{11} & \cdots & d Z_{1 g} \\
\vdots & \ddots & \vdots \\
d Z_{h 1} & \cdots & d Z_{h g}
\end{array}\right), \quad \frac{\partial}{\partial Z}=\left(\begin{array}{ccc}
\frac{\partial}{\partial Z_{11}} & \cdots & \frac{\partial}{\partial Z_{h 1}} \\
\vdots & \ddots & \vdots \\
\frac{\partial}{\partial Z_{1 g}} & \cdots & \frac{\partial}{\partial Z_{h g}}
\end{array}\right)
$$

Then an easy computation yields

$$
\begin{aligned}
& \frac{\partial}{\partial \lambda}=\Omega \frac{\partial}{\partial Z}+\bar{\Omega} \frac{\partial}{\partial \bar{Z}}, \\
& \frac{\partial}{\partial \mu}=\frac{\partial}{\partial Z}+\frac{\partial}{\partial \bar{Z}} .
\end{aligned}
$$

Thus we obtain the following

$$
\frac{\partial}{\partial \bar{Z}}=\frac{i}{2}(\operatorname{Im} \Omega)^{-1}\left(\frac{\partial}{\partial \lambda}-\Omega \frac{\partial}{\partial \mu}\right) .
$$

LEMMA 2.1.

$$
\begin{aligned}
\Phi_{\Omega}^{(\mu)} & {\left[\begin{array}{c}
A \\
0
\end{array}\right](\Omega \mid[(\lambda, \mu), \kappa]) } \\
& =\exp \left\{\pi i \sigma\left(\mathscr{M}\left(\lambda \Omega^{t} \lambda+\lambda^{t} \mu+\kappa\right)\right)\right\} \vartheta_{J}^{(\mu)}\left[\begin{array}{c}
A \\
0
\end{array}\right](\Omega \mid \lambda, \mu+\lambda \Omega) .
\end{aligned}
$$

Proof. It follows immediately from (1.4) and (1.5). 
LeMma 2.2. Let $\Phi([(\lambda, \mu), \kappa])$ be a real analytic function on $H_{Z}^{(g, h)} \backslash$ $H_{R}^{(g, h)}$ such that

i) $\exp \left\{-\pi i \sigma\left(\mathscr{M}_{\kappa}\right)\right\} \Phi([(\lambda, \mu), \kappa])$ is independent of $\kappa$,

ii) $\left(D_{m p}-\sum_{q=1}^{g} \Omega_{p q} \hat{D}_{m q}\right) \Phi=0$ for all $1 \leq m \leq h$ and $1 \leq p \leq g$, where $\mathscr{M}$ is a positive definite symmetric even integral matrix of degree $h$. Let

$$
\Psi(\lambda, \mu)=\exp \left\{-\pi i \sigma\left(\mathscr{M}\left(\lambda \Omega^{t} \lambda+\lambda^{t} \lambda+\kappa\right)\right)\right\} \Phi([(\lambda, \mu), \kappa]) .
$$

Then $\Psi(\lambda, \mu)$ is a mixed theta function of level $\mathscr{M}$ in $Z=\mu+\lambda \Omega$ with respect to $\Omega$.

Proof. By the assumption (i), we have

$$
\begin{aligned}
& \Psi(\lambda+\tilde{\lambda}, \mu+\tilde{\mu}) \\
& =\exp \left\{-\pi i \sigma\left(\mathscr{M}\left((\lambda+\tilde{\lambda}) \Omega^{t}(\lambda+\tilde{\lambda})+(\lambda+\tilde{\lambda})^{t}(\mu+\tilde{\mu})+\kappa+\tilde{\kappa}+\tilde{\lambda}^{t} \mu-\tilde{\mu}^{t} \lambda\right)\right)\right\} \\
& \Phi([(\tilde{\lambda}, \tilde{\mu}), \tilde{\kappa}] \circ[(\lambda, \mu), \kappa]) \\
& \quad=\exp \left\{-\pi i \sigma\left(\mathscr{M}\left(\tilde{\lambda} \Omega{ }^{t} \tilde{\lambda}+2(\mu+\lambda \Omega){ }^{t} \tilde{\lambda}\right)\right)\right\} \Psi(\lambda, \mu)
\end{aligned}
$$

where $[(\tilde{\lambda}, \tilde{\mu}), \tilde{\kappa}] \in H_{Z}^{(g, h)}$. In the last equality, we used the facts that $\sigma\left(\mathscr{M}\left(\tilde{\kappa}+\tilde{\lambda}^{t} \tilde{\mu}\right)\right) \in 2 Z$ because $\tilde{\kappa}+\tilde{\mu}^{t} \tilde{\lambda}$ is symmetric. This implies that $\Psi(\lambda, \mu)$ satisfies the condition (2) in Definition 1.2. Now we must show that $\Psi(\lambda, \mu)$ is holomorphic in $Z=\mu+\lambda \Omega$, that is,

$$
\frac{\partial \Psi}{\partial \bar{Z}}=0, \quad Z=\mu+\lambda \Omega
$$

By (2.2) the equation (2.4) is equivalent to the equation

$$
\left(\frac{\partial}{\partial \lambda_{m p}}-\sum_{q=1}^{g} \Omega_{p q} \frac{\partial}{\partial \mu_{m q}}\right) \Psi(\lambda, \mu)=0, \quad 1 \leq m \leq h, \quad 1 \leq p \leq g .
$$

But according to (1.9) and (1.10), we have

$$
\frac{\partial}{\partial \lambda_{m p}}-\sum_{q=1}^{g} \Omega_{p q} \frac{\partial}{\partial \mu_{m q}}=D_{m p}-\sum_{q=1}^{g} \Omega_{p q} \hat{D}_{m q}+P,
$$

where

$$
\begin{aligned}
P= & \sum_{k=1}^{m} \mu_{k p} D_{k m}^{0}+\sum_{k=m+1}^{h} \mu_{k p} D_{m k}^{0}-\sum_{k=1}^{m} \sum_{q=1}^{g} \Omega_{p q} \lambda_{k q} D_{k m}^{0} \\
& -\sum_{k=m+1}^{h} \sum_{q=1}^{g} \Omega_{p q} \lambda_{k q} D_{m k}^{0} .
\end{aligned}
$$

We observe that $P \cdot \Psi(\lambda, \mu)=0$ because $\Psi(\lambda, \mu)$ is independent of $\kappa$ by the assumption (i). We let 


$$
f([(\lambda, \mu), \kappa])=\exp \left\{-\pi i \sigma\left(\mathscr{M}\left(\lambda \Omega^{t} \lambda+\lambda^{t} \mu+\kappa\right)\right)\right\} .
$$

Then $\Psi(\lambda, \mu)=f([(\lambda, \mu), \kappa]) \Phi([(\lambda, \mu), \kappa])$. Then in order to show that $\Psi(\lambda, \mu)$ is holomorphic in the complex variables $Z=\mu+\lambda \Omega$ with respect to $\Omega$, by the assumption (ii), it suffices to show the following:

$$
\left(D_{m p}-\sum_{q=1}^{g} \Omega_{p q} \hat{D}_{m q}\right) f([(\lambda, \mu), \kappa])=0 .
$$

By an easy computation, we obtain (2.6). This completes the proof of Lemma 2.2.

q.e.d.

The Stone-von Neumann theorem says that an irreducible representation $\rho_{c}$ of $H_{R}^{(g, h)}$ is characterized by a real symmetric matrix $c \in R^{(h, h)}(c \neq 0)$ such that

$$
\rho_{c}([(\lambda, \mu), \kappa])=\exp \{\pi i \sigma(c \kappa)\} I, \quad \kappa={ }^{t} \kappa \in R^{(h, h)},
$$

where $I$ denotes the identity map of the representation space. If $c=0$, it is characterized by a pair $(k, m) \in R^{(h, g)} \times R^{(h, g)}$ such that

$$
\rho_{k, m}([(\lambda, \mu), \kappa])=\exp \left\{2 \pi i \sigma\left(k^{t} \lambda+m^{t} \mu\right)\right\} I .
$$

If $\Phi \in L^{2}\left(H_{Z}^{(g, h)} \backslash H_{R}^{(g, h)}\right)$ and $\tilde{\kappa}={ }^{t} \tilde{\kappa} \in Z^{(h, h)}$, then

$$
\begin{aligned}
\Phi([(\lambda, \mu), \kappa]) & =\Phi([(0,0), \tilde{\kappa}] \circ[(\lambda, \mu), \kappa]) \\
& =\Phi([(\lambda, \mu), \kappa] \circ[(0,0), \tilde{\kappa}]) \\
& =\rho_{c}([(0,0), \tilde{\kappa}]) \Phi([(\lambda, \mu), \kappa]) \\
& =\exp \{\pi i \sigma(c \tilde{\kappa})\} \Phi([(\lambda, \mu), \kappa]) .
\end{aligned}
$$

Thus if $c \neq 0, \sigma(c \tilde{\kappa}) \in 2 Z$ for all $\tilde{\kappa}={ }^{t} \tilde{\kappa} \in Z^{(h, h)}$. It means that ${ }^{t} c=c=\left(c_{i j}\right)$ must be even integral, that is, all diagonal elements $c_{i i}(1 \leq i \leq h)$ are even integers and all $c_{i j}(i \neq j)$ are integers. If $c=0, \sigma\left(k^{t} \lambda+m^{t} \mu\right) \in Z$ for all $\lambda, \mu \in Z^{(h, g)}$ and hence $k, m \in Z^{(h, g)}$. Therefore only the irreducible representation $\rho_{\mathscr{K}}$ with $\mathscr{M}={ }^{t} \mathscr{M}$ even integral and $\rho_{k, m}\left(k, m \in Z^{(h, g)}\right)$ could occur in the right regular representation $\rho$ in $L^{2}\left(H_{Z}^{(g, h)} \backslash H_{R}^{(g, h)}\right)$.

Now we prove

MaIN Theorem. Let $\mathscr{N} \neq 0$ be an even integral matrix of degree $h$ which is neither positive nor negative definite. Let $R(\mathscr{N})$ be the sum of irreducible representations $\rho_{\mathscr{r}}$ which occur in the right regular representation $\rho$ of $H_{R}^{(g, h)}$. Let $H_{\Omega}^{(\alpha)}\left[\begin{array}{c}A \\ 0\end{array}\right]$ be defined in Theorem 2 for a positive definite even integral matrix $\mathscr{M}>0$. Then the decomposition of the right regular representation $\rho$ is given by 


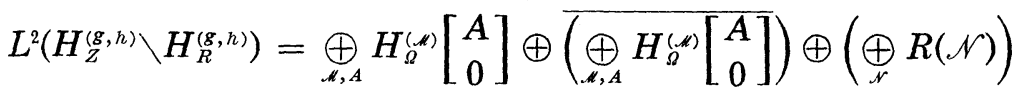

$$
\begin{aligned}
& \oplus\left(\underset{(k, m) \in Z^{(h, g)}}{\oplus} C \exp \left\{2 \pi i \sigma\left(k^{t} \lambda+m^{t} \mu\right)\right\}\right) \text {. }
\end{aligned}
$$

where $\mathscr{M}($ resp. $\mathscr{N})$ runs over the set of all positive definite symmetric, even integral matrices of degree $h$ (resp. the set of all even integral nonzero matrices of degree $h$ which are neither positive nor negative definite) and $A$ runs over a complete system of representatives of the cosets $\mathscr{M}^{-1} Z^{(h, g)} / Z^{(h, g)}$.

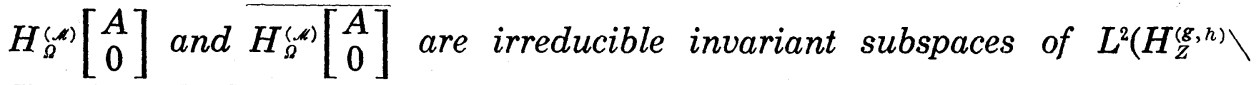
$H_{R}^{(g, h)}$ ) such that

$$
\begin{aligned}
& \rho([(0,0), \tilde{\kappa}]) \phi([(\lambda, \mu), \kappa])=\exp \{\pi i \sigma(\mathscr{M} \tilde{\kappa})\} \phi([(\lambda, \mu), \kappa]), \\
& \rho([(0,0), \tilde{\kappa}]) \overline{\phi([(\lambda, \mu), \kappa])}=\exp \{-\pi i \sigma(\mathscr{M} \tilde{\kappa})\} \overline{\phi([(\lambda, \mu), \kappa])}
\end{aligned}
$$

for all $\phi \in H_{\Omega}^{(\Omega)}\left[\begin{array}{c}A \\ 0\end{array}\right]$. And we have

$$
H_{\Omega}^{(\leftrightarrow)}\left[\begin{array}{c}
A \\
0
\end{array}\right]=\exp \left\{2 \pi i \sigma\left(\mathscr{M} \mu^{t} A\right)\right\} H_{\Omega}^{(\alpha)}\left[\begin{array}{l}
0 \\
0
\end{array}\right]
$$

This result generalizes that of $H$. Morikawa ([M]).

Proof. Let $\mathscr{A}$ be the space of real analytic functions on $L^{2}\left(H_{Z}^{(g, h)} \backslash\right.$ $\left.H_{R}^{(g, h)}\right)$. Since $\mathscr{A}$ is dense in $L^{2}\left(H_{Z}^{(g, h)} \backslash H_{R}^{(g, h)}\right)$ and $\mathscr{A}$ is invariant under $\rho$, it suffices to decompose $\mathscr{A}$. Let $W$ be an irreducible invariant subspace of $\mathscr{A}$ such that $\rho([(0,0), \tilde{\kappa}]) w=\exp \{2 \pi i \sigma(\mathscr{M} \tilde{\kappa})\} w$ for all $w \in W$, where $\mathscr{M}=$ ${ }^{t} \mathscr{M}$ is a positive definite even integral matrix of degree $h$. Then $W$ is isomorphic to $H_{\Omega}^{(k)}\left[\begin{array}{c}A \\ 0\end{array}\right] \cap \mathscr{A}$ for some $A \in \mathscr{M}^{-1} Z^{(h, g)} / Z^{(h, g)}$ and $\Omega \in H_{g}$. Since $H_{\Omega}^{(\mu)}\left[\begin{array}{c}A \\ 0\end{array}\right] \cap \mathscr{A}$ contains an element $\Phi_{0}^{(\mu)}\left[\begin{array}{c}A \\ 0\end{array}\right](\Omega \mid[(\lambda, \mu), \kappa]$ ) (see Corollary 1.4) satisfying

$$
\left(D_{m p}-\sum_{q=1}^{g} \Omega_{p q} \hat{D}_{m q}\right) \Phi_{0}^{(\cdot)}\left[\begin{array}{c}
A \\
0
\end{array}\right](\Omega \mid[(\lambda, \mu), \kappa])=0
$$

for all $1 \leq m \leq h, 1 \leq p \leq g$, there exists an element $\Phi_{0}([(\lambda, \mu), \kappa])$ in $W$ such that

$$
\left(D_{m p}-\sum_{q=1}^{g} \Omega_{p q} \hat{D}_{m q}\right) \Phi_{0}([(\lambda, \mu), \kappa])=0
$$

for all $1 \leq m \leq h, 1 \leq p \leq g$. On the other hand, we have 


$$
\begin{aligned}
\Phi_{0}([(\lambda, \mu), \kappa]) & =\rho([(0,0), \kappa]) \Phi_{0}([(\lambda, \mu), 0]) \\
& =\exp \{\pi i \sigma(\mathscr{M} \kappa)\} \Phi_{0}([(\lambda, \mu), 0]) .
\end{aligned}
$$

Therefore $\Phi_{0}([(\lambda, \mu), \kappa])$ satisfies the conditions of Lemma 2. Thus we have

$$
\begin{aligned}
\Phi_{0}([(\lambda, \mu), \kappa]) & =\exp \left\{\pi i \sigma\left(\mathscr{M}\left(\lambda \Omega^{t} \lambda+\lambda^{t} \mu+\kappa\right)\right)\right\} \sum_{A, J} \alpha_{A J} \vartheta_{J}^{(\mu)}\left[\begin{array}{c}
A \\
0
\end{array}\right](\Omega \mid \lambda, \mu+\lambda \Omega) \\
& =\sum_{A, J} \alpha_{A J} \Phi_{J}^{(\mu)}\left[\begin{array}{c}
A \\
0
\end{array}\right](\Omega \mid[(\lambda, \mu), \kappa]) \quad \text { (by Lemma 2.1) }
\end{aligned}
$$

where $A$ (resp. $J$ ) runs over $\mathscr{M}^{-1} Z^{(h, g)} / Z^{(h, g)}$ (resp. $Z_{\geq 0}^{(h, g)}$ ). Hence $\Phi_{0} \in$ $\oplus_{A} H_{\Omega}^{(k)}\left[\begin{array}{c}A \\ 0\end{array}\right]$. By the way, since $W$ is spanned by $D_{k l}^{0} \Phi_{0}, D_{m p} \Phi_{0}$ and $\hat{D}_{m p} \Phi_{0}$, we have $W \subset \oplus_{A} H_{\Omega}^{(k)}\left[\begin{array}{c}A \\ 0\end{array}\right] . \quad$ So $W=H_{\Omega}^{(\cdot)}\left[\begin{array}{c}A \\ 0\end{array}\right] \cap \mathscr{A} \quad$ for $\quad$ some $\quad A \in$ $\mathscr{M}^{-1} Z^{(h, g)} / Z^{(h, g)}$. Similarly, $\bar{W}=\overline{H_{\Omega}^{(\mathscr{k})}\left[\begin{array}{c}A \\ 0\end{array}\right]} \cap \mathscr{A}$. Clearly for each $(k, m) \in$ $Z^{(h, g)} \times Z^{(h, g)}$,

$$
W_{k, m}:=C \exp \left\{2 \pi i\left(k^{t} \lambda+m^{t} \mu\right)\right\}
$$

is a one dimensional irreducible invariant subspace of $L^{2}\left(H_{Z}^{(h, g)} \backslash H_{R}^{(g, h)}\right)$. The latter part of the above theorem is the restatement of Theorem 2. This completes the main theorem.

q.e.d.

Corollary. For even integral matrix $\mathscr{M}={ }^{t} \mathscr{M}>0$ of degree $h$, the multiplicity $m_{\mathscr{k}}$ of $\rho_{\mathscr{A}}$ in $\rho$ is given by

$$
m_{\mathscr{M}}=(\operatorname{det} \mathscr{M})^{g} \text {. }
$$

Conjecture. For any even integral matrix $\mathscr{N} \neq 0$ of degree $h$ which is neither positive nor negative definite, the multiplicity $m_{\mathscr{r}}$ of $\rho_{\mathscr{r}}$ in $\rho$ is a zero, that is, $R(\mathscr{N})$ vanishes.

\section{§3. Schrödinger representations}

Let $\Omega \in H_{g}$ and let $\mathscr{M}={ }^{t} \mathscr{M}$ be a positive definite even integral matrix of degree $h$. We set $\Omega=\Omega_{1}+i \Omega_{2}\left(\Omega_{1}, \Omega_{2} \in R^{(g, g)}\right)$. Let $L^{2}\left(R^{(h, g)}, \mu_{\Omega_{2}}^{(k)}\right)$ be the $L^{2}$-space of $R^{(h, g)}$ with respect to the measure

$$
\mu_{\Omega_{2}}^{(\mathscr{A})}(d \xi)=\exp \left\{-2 \pi \sigma\left(\mathscr{M} \xi \Omega_{2}{ }^{t} \xi\right)\right\} d \xi
$$

It is easy to show that the transformation $f(\xi) \mapsto \exp \left\{\pi i \sigma\left(\mathscr{M} \xi \Omega_{2}{ }^{t} \xi\right)\right\} f(\xi)$ of $L^{2}\left(R^{(h, g)}, \mu_{\Omega_{2}}^{(\mathcal{k})}\right)$ into $L^{2}\left(R^{(h, g)}, d \xi\right)$ is an isomorphism. Since the set $\left\{\xi^{J} \mid J \in\right.$ $\left.Z_{\geq 0}^{(h, g)}\right\}$ is a basis of $L^{2}\left(R^{(h, g)}, \mu_{\Omega_{2}}^{(\mu)}\right)$, the set $\left\{\exp \left(\pi i \sigma\left(\mathscr{M} \xi \Omega^{t} \xi\right)\right) \xi^{J} \mid J \in Z_{\geq 0}^{(h, g)}\right\}$ is a basis of $L^{2}\left(R^{(h, g)}, d \xi\right)$. 
LEMMA 3.1.

$$
\begin{aligned}
& \left\langle\Phi_{J}^{(\mathscr{\mu})}\left[\begin{array}{c}
A \\
0
\end{array}\right](\Omega \mid[(\lambda, \mu), \kappa]), \Phi_{K}^{(\tilde{\mu})}\left[\begin{array}{c}
\tilde{A} \\
0
\end{array}\right](\Omega \mid[(\lambda, \mu), \kappa])\right\rangle \\
& =\int_{H_{Z}^{(g, h)} \backslash H_{R}^{(g, h)}} \Phi_{J}^{(\mu)}\left[\begin{array}{c}
A \\
0
\end{array}\right](\Omega \mid[(\lambda, \mu), \kappa]) \cdot \overline{\left.\Phi_{K}^{(\tilde{\mu})}\left[\begin{array}{c}
A \\
0
\end{array}\right](\Omega \mid[\lambda, \mu), \kappa]\right)} d \lambda d \mu d \kappa \\
& =\left\{\begin{array}{l}
\int_{R^{(h, g)}} y^{J+K} \exp \left\{-2 \pi \sigma\left(\mathscr{M} y \Omega_{2}{ }^{t} y\right)\right\} d y \quad \text { if } \mathscr{M}=\tilde{\mathscr{M}}, A \equiv \tilde{A}(\bmod \mathscr{M}), \\
0, \quad \text { otherwise. }
\end{array}\right.
\end{aligned}
$$

It is easy to prove the above lemma and so we omit its proof. According to the above argument and Lemma 3.1, we obtain the following: by

$$
\xi^{J} \longmapsto \Phi_{J}^{(\alpha)}\left[\begin{array}{c}
A \\
0
\end{array}\right](\Omega \mid[(\lambda, \mu), \kappa]), \quad J \in Z_{\geq 0}^{(h, g)}
$$

is an isomorphism of Hilbert spaces.

Now we define a unitary representation of $H_{R}^{(h, g)}$ on $L^{2}\left(R^{(h, g)}, d \xi\right)$ by

$$
U_{\mathscr{M}}([(\lambda, \mu), \kappa]) f(\xi)=\exp \left\{-\pi i \sigma\left(\mathscr{M}\left(\kappa+\mu^{t} \lambda+2 \mu^{t} \xi\right)\right)\right\} f(\xi+\lambda),
$$

where $[(\lambda, \mu), \kappa] \in H_{R}^{(g, h)}$ and $f \in L^{2}\left(R^{(h, g)}, d \xi\right) . \quad U_{\|}$is called the Schrödinger representation of $H_{R}^{(h, g)}$ of index $\mathscr{M}$.

Proposition 3.3. If we set $f_{J}(\xi)=\exp \left\{\pi i \sigma\left(\mathscr{M} \xi \Omega^{t} \xi\right)\right\} \xi^{J}\left(J \in Z_{\geq 0}^{(h, g)}\right)$, we have

$$
\begin{aligned}
& d U_{\mathscr{M}}\left(D_{k l}^{0}\right) f_{J}(\xi)=-\pi i \mathscr{M}_{k l} f_{J}(\xi), \quad 1 \leq k \leq l \leq h \\
& d U_{\mathscr{M}}\left(D_{m p}\right) f_{J}(\xi)=2 \pi i \sum_{l=1}^{h} \sum_{q=1}^{g} \mathscr{M}_{m l} \Omega_{p q} f_{J+\varepsilon_{l q}}(\xi)+J_{m p} f_{J-\varepsilon_{m p}}(\xi) . \\
& d U_{\mathscr{M}}\left(\hat{D}_{m p}\right) f_{J}(\xi)=-\pi i \sum_{l=1}^{h} \mathscr{M}_{m l} f_{J+\varepsilon_{l p}}(\xi) .
\end{aligned}
$$

Proof.

$$
\begin{aligned}
d U_{\mathcal{M}}\left(D_{k l}^{0}\right) f_{J}(\xi) & =\left.\frac{d}{d t}\right|_{t=0} U_{\mathscr{M}}\left(\exp \left(t X_{k l}^{0}\right)\right) f_{J}(\xi) \\
& =\left.\frac{d}{d t}\right|_{t=0} U_{\mathcal{M}}\left(\left[(0,0), t E_{k l}^{0}\right]\right)_{J}(\xi) \\
& =\lim _{t \rightarrow 0} \frac{\exp \left\{-\pi i \sigma\left(t \mathscr{M} E_{k l}^{0}\right)\right\}-I}{t} f_{J}(\xi) \\
& =-\pi i \mathscr{M}_{k l} f_{J}(\xi) .
\end{aligned}
$$




$$
\begin{aligned}
d U_{\mathscr{A}}\left(D_{m p}\right) f_{J}(\xi) & =\left.\frac{d}{d t}\right|_{t=0} U_{\mathscr{A}}\left(\exp \left(t X_{m p}\right)\right) f_{J}(\xi) \\
& =\left.\frac{d}{d t}\right|_{t=0} U_{\mathscr{M}}\left(\left[\left(t E_{m p}, 0\right), 0\right]\right) f_{J}(\xi) \\
& =\left.\frac{d}{d t}\right|_{t=0} \exp \left\{\pi i \sigma\left(\mathscr{M}\left(\xi+{ }^{t} E_{m p}\right) \Omega^{t}\left(\xi+t E_{m p}\right)\right)\right\}\left(\xi+t E_{m p}\right)^{J} \\
& =2 \pi i \sum_{l=1}^{h} \sum_{q=1}^{g} \mathscr{M}_{m l} \Omega_{p q} f_{J+\varepsilon_{l q}}(\xi)+J_{m p} f_{J-\varepsilon_{m p}}(\xi) .
\end{aligned}
$$

Finally,

$$
\begin{aligned}
d U_{\mathscr{M}}\left(\hat{D}_{m p}\right) f_{J}(\xi) & =\left.\frac{d}{d t}\right|_{t=0} U_{\mathscr{M}}\left(\exp \left(t \hat{X}_{m p}\right)\right) f_{J}(\xi) \\
& =\left.\frac{d}{d t}\right|_{t=0} U_{\mathscr{M}}\left(\left[\left(0, t E_{m p}\right), 0\right]\right) f_{J}(\xi) \\
& =\lim _{t \rightarrow 0} \frac{\exp \left\{-2 \pi i \sigma\left(t \mathscr{M} E_{m p}{ }^{t} \xi\right)\right\}-I}{t} f_{J}(\xi) \\
& =-\pi i \sum_{l=1}^{h} \mathscr{M}_{m l} f_{J+\varepsilon_{l p} p}(\xi) .
\end{aligned}
$$

q.e.d.

TheOREM 3. Let $\Phi_{\Omega}^{(k)}\left[\begin{array}{c}A \\ 0\end{array}\right]$ be the transform of $L^{2}\left(R^{(h, g)}, d \xi\right)$ onto $H_{\Omega}^{(u)}\left[\begin{array}{c}A \\ 0\end{array}\right]$ defined by

$$
\begin{aligned}
\Phi_{\Omega}^{(\mu)}\left[\begin{array}{c}
A \\
0
\end{array}\right]\left(\exp \left(\pi i \sigma\left(\mathscr{M} \xi \Omega^{t} \xi\right)\right) \xi^{J}\right)([(\lambda, \mu), \kappa]) \\
\quad=\Phi_{J}^{(\mu)}\left[\begin{array}{c}
A \\
0
\end{array}\right](\Omega \mid[(\lambda, \mu), \kappa]), \quad J \in Z_{\geq 0}^{(h, g)} .
\end{aligned}
$$

Then $\Phi_{\Omega}^{(\alpha)}\left[\begin{array}{c}A \\ 0\end{array}\right]$ is an isomorphism of the Hilbert space $L^{2}\left(R^{(h, g)}, d \xi\right)$ onto the Hilbert space $H_{\Omega}^{(.)}\left[\begin{array}{c}A \\ 0\end{array}\right]$ such that

$$
\begin{aligned}
& \hat{\rho}([(\lambda, \mu), \kappa]) \circ \Phi_{\Omega}^{(\alpha)}\left[\begin{array}{c}
A \\
0
\end{array}\right]=\Phi_{\Omega}^{(\alpha)}\left[\begin{array}{c}
A \\
0
\end{array}\right] \circ U_{\mathscr{A}}([(\lambda, \mu), \kappa]), \\
& \Phi_{\Omega}^{(\mathscr{k})}\left[\begin{array}{c}
A \\
0
\end{array}\right]=\exp \left\{2 \pi i \sigma\left(\mathscr{M} A^{t} \mu\right)\right\} \rho([(A, 0), 0]) \Phi_{\Omega}^{(\mu)}\left[\begin{array}{l}
0 \\
0
\end{array}\right],
\end{aligned}
$$

where $\hat{\rho}$ is the unitary representation of $H_{R}^{(g, h)}$ on $H_{\Omega}^{(k)}\left[\begin{array}{c}A \\ 0\end{array}\right]$ defined by

$$
\hat{\rho}([(\lambda, \mu), \kappa]) \phi=\rho([(\lambda,-\mu),-\kappa]) \phi, \quad \phi \in H_{\Omega}^{(\mu)}\left[\begin{array}{c}
A \\
0
\end{array}\right]
$$


Proof. For brevity, we set $f_{.}(\xi)=\exp \left\{\pi i \sigma\left(\mathscr{M} \xi \Omega{ }^{t} \xi\right)\right\} \xi^{J}\left(J \in Z_{\geq 0}^{(h, g)}\right)$. Using Proposition 3.3, we obtain

$$
\begin{aligned}
& \Phi_{a}^{(\not)}\left[\begin{array}{c}
A \\
0
\end{array}\right]\left(d U_{\mathscr{A}}\left(-D_{k i}^{0}\right)\left(f_{J}(\xi)\right)\right)([(\lambda, \mu), \kappa]) \\
& =\pi i \mathscr{M}_{k l} \Phi_{\Omega}^{(\mu)}\left[\begin{array}{c}
A \\
0
\end{array}\right]\left(f_{J}(\xi)\right)([(\lambda, \mu), \kappa]) \\
& =\pi i \mathscr{M}_{k l} \Phi_{J}^{(k)}\left[\begin{array}{c}
A \\
0
\end{array}\right](\Omega \mid[(\lambda, \mu), \kappa]) \\
& =d \rho\left(D_{k l}^{0}\right)\left\{\Phi_{\Omega}^{(k)}\left[\begin{array}{c}
A \\
0
\end{array}\right]\left(f_{J}(\xi)\right)[(\lambda, \mu), \kappa]\right\} . \\
& \Phi_{\Omega}^{(\not)}\left[\begin{array}{c}
A \\
0
\end{array}\right]\left(d U_{\mathcal{M}}\left(D_{m p}\right)\left(f_{J}(\xi)\right)\right)([(\lambda, \mu), \kappa]) \\
& =2 \pi i \sum_{l=1}^{h} \sum_{q=1}^{g} \mathscr{M}_{m l} \Omega_{p q} \Phi_{\Omega}^{(\cdot k)}\left[\begin{array}{c}
A \\
0
\end{array}\right]\left(f_{J+\varepsilon_{l q}}(\xi)\right)([(\lambda, \mu), \kappa]) \\
& +J_{m p} \Phi_{a}^{(k)}\left[\begin{array}{c}
A \\
0
\end{array}\right]\left(f_{J-\varepsilon_{m p}}(\xi)\right)([(\lambda, \mu), \kappa]) \\
& =2 \pi i \sum_{l=1}^{h} \sum_{q=1}^{g} \mathscr{M}_{m l} \Omega_{p q} \Phi_{J+\varepsilon_{l q}}^{(k)}\left[\begin{array}{c}
A \\
0
\end{array}\right](\Omega \mid[(\lambda, \mu), \kappa]) \\
& +J_{m p} \Phi_{J-\varepsilon_{m p}(\mu)}^{(\lambda)}\left[\begin{array}{c}
A \\
0
\end{array}\right](\Omega \mid[(\lambda, \mu), \kappa]) \\
& =d \rho\left(D_{m p}\right) \Phi_{J}^{(k)}\left[\begin{array}{c}
A \\
0
\end{array}\right](\Omega \mid[(\lambda, \mu), \kappa]) \\
& =d \rho\left(D_{m p}\right)\left\{\Phi_{\Omega}^{(k)}\left[\begin{array}{c}
A \\
0
\end{array}\right]\left(f_{J}(\xi)\right)([(\lambda, \mu), \kappa])\right\} .
\end{aligned}
$$

Finally, we obtain

$$
\begin{aligned}
\Phi_{\Omega}^{(\mu)} & {\left[\begin{array}{c}
A \\
0
\end{array}\right]\left(d U_{\mathscr{A}}\left(-\hat{D}_{m p}\left(f_{J}(\xi)\right)\right)([(\lambda, \mu), \kappa])\right.} \\
& =\pi i \sum_{l=1}^{h} \mathscr{M}_{m l} \Phi_{\Omega}^{(\mu)}\left[\begin{array}{c}
A \\
0
\end{array}\right]\left(f_{J+\varepsilon_{l} p}(\xi)\right)([(\lambda, \mu), \kappa]) \\
& =\pi i \sum_{l=1}^{h} \mathscr{M}_{m l} \Phi_{J+\varepsilon_{l} p}^{(\mu)}\left[\begin{array}{c}
A \\
0
\end{array}\right](\Omega \mid[(\lambda, \mu), \kappa]) \\
& =d \rho\left(\hat{D}_{m p}\right) \Phi_{J}^{(\mu)}\left[\begin{array}{c}
A \\
0
\end{array}\right](\Omega \mid[(\lambda, \mu), \kappa]) \\
& =d \rho\left(\hat{D}_{m p}\right)\left\{\Phi_{\Omega}^{(\alpha)}\left[\begin{array}{c}
A \\
0
\end{array}\right]\left(f_{J}(\xi)\right)([(\lambda, \mu), \kappa])\right\},
\end{aligned}
$$

where $1 \leq k \leq l \leq h, 1 \leq p \leq g$. The last statement is obvious. q.e.d. 
Remark 3.4. Theorem 3 means that the unitary representation $\hat{\rho}$ of $H_{R}^{(g, h)}$ on $H_{\Omega}^{(k)}\left[\begin{array}{c}A \\ 0\end{array}\right]$ is equivalent to the Schrödinger representation $U_{\mathcal{K}}$ of index $\mathscr{M}$. Thus the Schrödinger representation $U_{*}$ is irreducible.

\section{REFERENCES}

[C] P. Cartier, Quantum Mechanical Commutation Relations and Theta Functions, Proc. of Symposia in Pure Mathematics, A.M.S., 9 (1966), 361-383.

[I] J. Igusa, Theta functions, Springer-Verlag (1972).

[M] H. Morikawa, Some results on harmonic analysis on compact quotients of Heisenberg groups, Nagoya Math. J., 99 (1985), 45-62.

[T] M. Taylor, Noncommutative Harmonic Analysis, Math. Surveys and Monographs, Amer. Math. Soc., No. 22 (1986).

[Wei] A. Weil, Sur certains groupes d'operateurs unitaires, Acta Math., 113 (1964), 143-211.

[Wey] H. Weyl, The theory of groups and quantum mechanics, Dover Publications, New York (1950).

[Z] C. Ziegler, Jacobi Forms of Higher Degree, Abh. Math. Sem. Univ. Hamburg, 59 (1989), 191-224.

Department of Mathematics

Inha University

Incheon, 402-752

Republic of Korea 